\title{
Design of a magnetic actuation system for a microbiota-collection ingestible capsule
}

\author{
Martina Finocchiaro, Cristina Giosuè, Gaspare Drago, Fabio Cibella, Arianna Menciassi, Member \\ IEEE, Mario Sprovieri, Gastone Ciuti, Member IEEE
}

\begin{abstract}
Minimally invasive wireless devices, allowing the sampling of gut's bacteria, are needed for a longitudinal understanding of the role of the microbiota on the human health. Herein, we present a novel magnetic actuation system fitting inside a $11.5 \times 30.5 \mathrm{~mm}$ wireless ingestible capsule. Lacking any electronic components, the capsule robot is designed for the collection of microbiota's samples through mechanical brushing. Wireless activation and in situ sampling are enabled by an external permanent magnetic source. This component, when approaching the capsule, progressively allows: (1) the adhesion of the device to the mucosa, (2) the exposure of the brushes, and (3) the sampling by multiple rotations. Numerical and analytical models were developed for dimensioning the system, and were validated by benchtop experiments.
\end{abstract}

\section{INTRODUCTION}

In the last decade, substantial research has been investigating the relationship between the microbiota/microbiome and the human health [1]. By definition, the microbiota is the collection of 10-100 trillion microbial cells living in symbiosis with the human body, primarily, i.e. $>90 \%$, in the gastrointestinal (GI) tract [2]. As shown by multiple studies, an alteration of the gut microbial composition (i.e., dysbiosis) is associated with pathogenesis of several diseases, e.g. diabetes, obesity, inflammatory bowel diseases, cardio-vascular and also psychological disorders [1]. However, whether the dysbiosis is a cause, a consequence, or a modulator of the disease has not been carefully investigated yet [3]. One of the main reasons for this research gap, is due to the current invasive techniques for sampling the microbiota (e.g., biopsy or mucosal brushing during endoscopic procedures), hence limited only to symptomatic patients. As a solution, the development of noninvasive devices, such as ingestible wireless capsules, would make the sampling of microbiota more accessible, including

* This work was supported by the ATLAS project. This project has received funding from the European Union's Horizon 2020 research and innovation programme under the Marie Sklodowska-Curie grant agreement No 813782.This work was also part of the CISAS (MIUR-CNR) project, funded by the Italian Ministry of University and Research.

M. Finocchiaro, A. Menciassi and G. Ciuti are with the BioRobotics Institute, Scuola Superiore Sant'Anna, Italy and with the Department of Excellence in Robotics \& AI, Scuola Superiore Sant'Anna, Italy. M. Finocchiaro is also with Center of Research in Biomedical Engineering of Universitat Politècnica de Catalunya, Spain (corresponding author's e-mail: martina.finocchiaro@upc.edu).

M. Sprovieri and C. Giosuè are with National Research Council (CNR), Institute of Anthropic Impacts and Sustainability in the Marine Environment (IAS), Italy.

G. Drago and F. Cibella are with National Research Council (CNR), Institute for Biomedical Research and Innovation (IRIB), Italy. the small bowel, which is so far not feasible by commonly used technologies. As a matter of fact, this alternative would have a massive impact in clinical research, as well as in future multi-modality and in situ diagnostic techniques [4].

In recent years, few endoscopic capsules for microbiota collection have been investigated. Salem et al. developed an ingestible device, whose opening is regulated by the dissolution of a bio-gradable coating, allowing the entrance of the fluid inside the capsule. At the same time, the closing is enabled by a passive bistable mechanism, triggered by volume increase of a sponge. Although promising, this solution does not enable choosing the specific location of collection, neither localizing the sample, and it does not consider the quality, quantity and correct storage of the material in order to properly analyze its composition through the bacterial 16S rRNA sequencing [5]. A more advanced solution is presented by Rezaei et al., who collected the bacteria through an osmotic pump, enabled by the dissolution of an external coating. In this case, it was also possible to successfully analyze the bacteria DNA' samples, collected in in-vivo conditions. Nonetheless, a spatially targeted sampling is still not feasible in this scenario, and the authors expressed their intention to use a magnetic system, in the future, to cover this gap [6].

In this framework, the current paper presents a novel magnetic actuation system for a microbiota collection capsule. The device, free of any electronical components, is designed to allow a safe mucosa brushing and sample storage. In situ wireless activation is enabled by the use of an external permanent magnet, which triggers the rotation of miniaturized magnets inside the capsule. Starting from the system requirements, the paper shows the design flow of the actuation system. In this context, a modular framework for the computation of the magnetic torques and forces enabling the actuation is presented. Finally, preliminary validation of the design, through numerical and analytical methods and with benchtop experiments, is reported.

\section{REQUIREMENTS AND CAPSULE CONCEPT}

\section{A. System requirements}

As an ingestible device, the endoscopic capsule needs to satisfy several limiting requirements, especially in terms of size and safety. Moreover, additional constraints are posed by the need of collecting and safely storing microbiota samples. Here, we list the main requirements that need to be addressed:

- Capsule dimensions lower or equal to those of the PillCam $^{\mathrm{TM}}$ (Medtronics, Minneapolis, USA), FDA approved ingestible device [7] - length of $32 \mathrm{~mm}$ and diameter of $12 \mathrm{~mm}$. 
- Localization and wireless activation of the sampling process in specific and selected locations.

- Safety of the device, avoiding protruding parts, loss of components or electromagnetic-associated risks.

- Volume of the collected sample sufficient for RNA sequencing analysis - equal or higher to $18 \mathrm{mg}$ [8].

- Appropriate storage of the collected sample inside the capsule. In current clinical practise, the microbiota sample is stored in a RNAlater ${ }^{\mathrm{TM}}$ stabilization solution to avoid degradation, right after the collection $\left(1: 5=\right.$ volumes of RNA later ${ }^{\mathrm{TM}}$ : tissue). Parallel researches are investigating the need of the preserving solution right after collection of the sample, or if it can be added few hours later, when the capsule is expulsed [9].

- Collection of at least two simultaneous samples for region of interest to enable a more robust analysis.

\section{B. Capsule concept}

Given the small volume available inside a pill-size ingestible capsule (i.e., around $2 \mathrm{~mL}$ ), the use of standard mechatronic systems (i.e., motors, drivers, electronic boards, batteries) poses several challenges. Moreover, safety issues related to the ingestion of electrical components impose additional limitations. As a solution, our design is built upon an actuation system made of only passive components, operated remotely by an external permanent magnet. As shown in Fig. 1, the mechanism fits inside a 11.5 x $30.5 \mathrm{~mm}$ capsule and allows the microbiota sampling through luminal brushing. This clinical technique provides the best proportion of bacteria to host DNA with respect to the other sampling methods, without involving any bleeding [9]. In our case, the specimen is collected with the capsule using two rotating brushes (Fig. 1.d-e). Each component has an external surface with comparable dimension (i.e., $50 \mathrm{~mm}^{2}$ ) to those used in clinics (i.e., Cook Medical Cythology brush). Each brush, once collected the tissue, stores it inside a separate reservoir, which is sized to house the correct volume of RNAlater ${ }^{\mathrm{TM}}$ (i.e., at least 5 times the volume of the sample). In fact, even though the mandatory use of a preservation solution is under investigation, authors decided to guarantee the needed space inside the capsule. Two gates cover the brushes; their opening, together with the sampling process, are controlled remotely (Fig. 1.d).

Finally, five $\mathrm{N} 52 \mathrm{NdFeB}$ internal permanent magnets (IPMs) constitutes the capsule's actuation system (Fig. 1.c-e). In particular, two of them have a hemispherical shape, and are fixed at the two ends of the capsule. Whereas two diametrically-magnetized hollow cylinders are mounted on a

TABLE 1. MAIN DESIGN SPECIFICATIONS

\begin{tabular}{|c|l|}
\hline Specifications & \multicolumn{1}{|c|}{ Values } \\
\hline Capsule dimension & $11.5 \times 30.5 \mathrm{~mm}$ \\
\hline Brush external surface & $50 \mathrm{~mm}^{2}$ each \\
\hline Reservoir size & $261 \mathrm{~mm}^{3}$ each \\
\hline Number of samples & 2 \\
\hline \multirow{3}{*}{ IPMs dimensions $[\mathrm{mm}]$} & $\mathrm{IPM}_{1,5}: \mathrm{R}=5.25$ \\
& $\mathrm{IPM}_{3}: \mathrm{D}=10, \mathrm{~d}=9, \mathrm{~L}=7$ \\
& $\mathrm{IPM}_{2,4}: \mathrm{D}=7, \mathrm{~d}=1.25, \mathrm{~L}=2$ \\
\hline
\end{tabular}
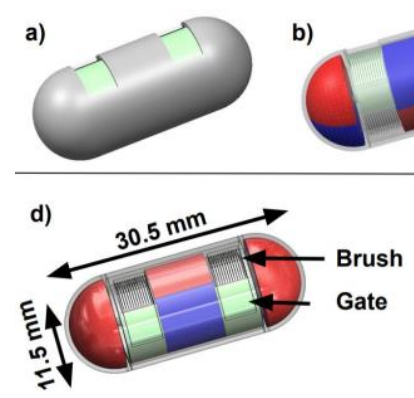

Figure 1.3D model of the endoscopic capsule: a) external view with closed gates; b) the capsule wall is removed to see the internal components; $c$ ) internal magnets arranged inside the capsule; d) the gates (in green), when opened, exposes the brushes (in grey) to the external environment; e) 3D

$\mathrm{CAD}$ of the five internal magnets and one of the two brushes.

shaft, and rigidly connected to the brushes, thus allowing their rotation. Finally, a third thin hollow diametricallymagnetized cylinder, free to rotate around the capsule internal wall, drives the opening and closing of the gates. A summary of the capsule's specifications is provided by Table 1 .

The IPMs rotations are driven by the variation of the magnetic field induced by an external permanent magnet (EPM). The system has three main working phases: (1) attraction of the capsule to the intestinal walls; (2) opening of the device; and (3) brushing of the mucosa. As Fig. 2 shows, when the EPM is placed at a distance $d_{l}$ from the capsule, the device is attracted to the mucosa by a force $F$. Once the EPM is moved to a distance $d_{2}$ smaller than $d_{l}$, the generated magnetic field forces the rotation of the gates, exposing the brushes. Lastly, when the EPM reaches a distance $d_{3}$ lower than $d_{2}$, the rotation of the cylindrical magnets is enabled, driving the brushing. In this case, the EPM is moved backward and forward of a $\Delta_{d}$ around $d_{3}$ (such that $d_{3}-\Delta_{d}<$ $d_{2}$ ), allowing multiple rotations of the brushes. Finally, when the sampling task has ended, the EPM is removed leading to the closure and releasing of the capsule.

\section{MAGNETIC ACTUATION SYSTEM}

\section{A. Magnetic principle}

The mechanism allowing the rotation of the gates and the brushes, is based on two non-linear magnetic torsional springs, firstly introduced by Simi et al. [10]. In fact, when placing two diametrically-magnetized hollow cylinders on a shaft, at fixed short distance, and free to rotate, they orient themselves to have parallel and opposite magnetization

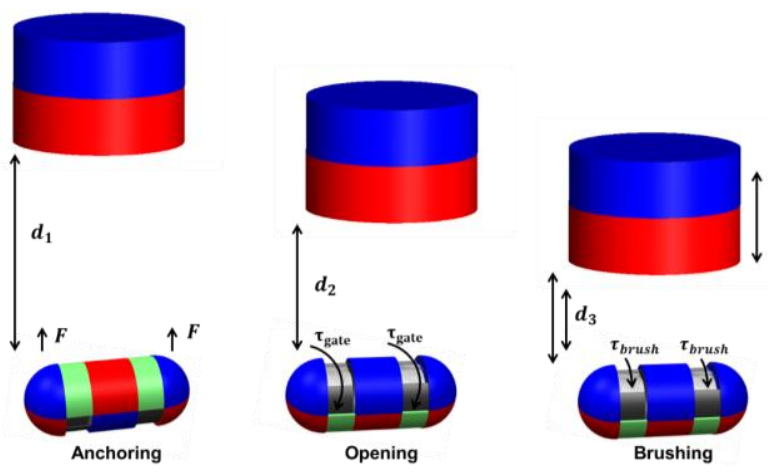

Figure 2. Operational flow: the EPM position triggers first the adhesion of the capsule to the intestinal walls, second the opening of the gates, and third the brushing of the mucosa. 
vectors. However, if only one of the two magnets is fixed, and an external magnetic field with opposite polarity is applied, strong enough to overcome the local magnetic coupling, the cylinder free to rotate will make a $180^{\circ}$ turn. Doing so, it will reach an unstable equilibrium point, and will store potential elastic energy. Once the external magnetic field is removed, the free magnet will go back to its initial position, releasing the stored energy. The main parameter that characterizes the magnetic torsional spring is the internal torque between the two magnets, when the angle between the corresponding magnetization vectors is $90^{\circ}$. This is the highest level of torque, named peak torque, that needs to be overcome by the external magnetic agent to rotate one of the two cylinders.

\section{B. Analytical description}

In our design, an extended version of the magnetic torsional spring is used to drive the system. More in details, referring to the nomenclature of Fig. 3, IPMs 1 and 5 work as the fixed magnets for three nested magnetic torsional springs (MTS). Accordingly, IPM $\mathrm{M}_{3}$ is the free-to-rotate element of the first MTS, allowing the opening of the gates. Whereas $\mathrm{IPM}_{2}$ and $\mathrm{IPM}_{4}$ are the rotational magnets of the other two MTSs.

In order to correctly dimension the system for guaranteeing the desired behavior, and create a scalable framework, a static model has been developed. Assuming that the environment does not contain any additional ferromagnetic material, it is possible to apply the principle of superposition for the computation of torques and forces induced by multiple magnets.

Fig. 3.a shows the orientation of the magnets in the rest position (i.e., gates closed and capsule free to navigate in the bowel). In this configuration, the internal torque exerted by the magnets fixed to the capsule (i.e., $\mathrm{IPM}_{1,5}$ ) on those free to rotate (i.e., $\mathrm{IPM}_{2,3,4}$ ), has to guarantee the stable closure of the capsule:

$$
\begin{aligned}
& \tau_{21}+\tau_{25}>\tau_{24}+\tau_{23} \\
& \tau_{31}+\tau_{35}>\tau_{32}+\tau_{34}
\end{aligned}
$$

Where, $\tau_{21}$ is the torque on $\mathrm{IPM}_{2}$ given by $\mathrm{IPM}_{1}, \tau_{25}$ is the torque on $\mathrm{IPM}_{2}$ given by $\mathrm{IPM}_{5}$, etc. Since the system is axially symmetric, the torque experienced by $\mathrm{IPM}_{2}$ is equal to the one of $\mathrm{IPM}_{4}$, and the same applies to torques on IPMs 1 and 5. Considering Fig. 3.a, as the first step towards the sampling process, the EPM placed at a distance $d_{l}$ from the capsule, need to generate an axial magnetic field gradient
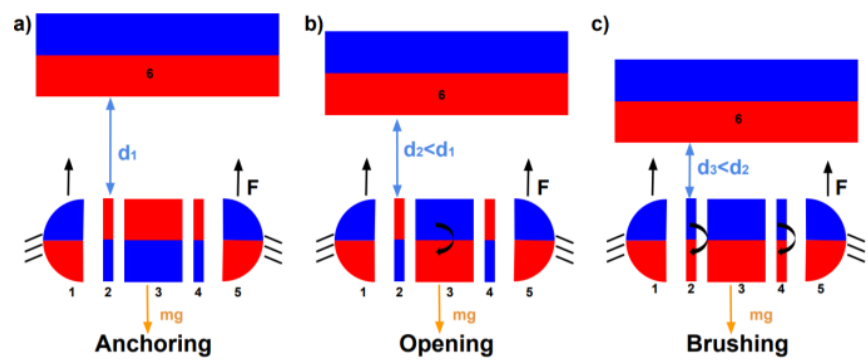

Figure 3. Diagram of the three operational phases and the polarization of the IPMs: a) capsule configuration in rest position, the EPM generate an attraction force on the IPMs; b) the EPM moves forward triggering the $\mathrm{IPM}_{3}$ rotation (first unstable equilibrium point); c) the EPM moves closer, enabling the rotation of $\mathrm{IPM}_{2}$ and $\mathrm{IPM}_{4}$ (second unstable equilibrium point) and the consequent brushing. such that:

$$
\begin{gathered}
\mathrm{F}\left(\mathrm{d}_{1}\right)=2 \mathrm{~F}_{1}-2 \mathrm{~F}_{2}-\mathrm{F}_{3}-\mathrm{mg}>0 \\
\mathrm{~F}_{1}=\mathrm{F}_{5}, \mathrm{~F}_{2}=\mathrm{F}_{4} .
\end{gathered}
$$

Where $F$ is the total vertical force exerted on the capsule, $F_{1}, F_{2}$ and $F_{3}$ refer respectively to the magnetic vertical force on IPM1,2,3 and $m g$ is the capsule weight force to overcome to ensure the adhesion of the system to the intestinal mucosa.

Secondly, placing the EPM closer to the capsule (Fig. 3.b), at a distance $d_{2}<d_{1}$, the torque induced by the EPM on $\mathrm{IPM}_{3}$ (i.e., $\tau_{36}$ ), must overcome the internal torques between $\mathrm{IPM}_{3}$ and the IPMs 1 and 5, thus allowing to open the gate:

$$
\tau_{36}\left(d_{2}\right)>2 \tau_{31}-2 \tau_{32} .
$$

At this stage, the brushes do not rotate yet, so the external magnetic field does not overcome the internal torques on IPMs 2 and 4:

$$
\tau_{26}\left(\mathrm{~d}_{2}\right)<\tau_{21}+\tau_{23}-\tau_{24}+\tau_{25}
$$

Finally, moving the EPM at a distance $d_{3}$ from the capsule (such that $d_{3}<d_{2}$ ), the external torque on IPMs 2 and 4 overcome the internal couplings, thus allowing the rotation of these two magnets (Fig. 3.c), while keeping the gates opened:

$$
\tau_{26}\left(\mathrm{~d}_{3}\right)>\tau_{21}+\tau_{23}-\tau_{24}+\tau_{25}
$$

\section{MAGNETIC MODELLING}

\section{A. Design workflow}

The dimensioning of the actuation system has involved multiple steps in order to meet the design requirements. Considering the maximum volume of the capsule and leaving space for the two reservoirs of RNAlater ${ }^{\mathrm{TM}}$, the internal magnets sizing is a trade-off between stability and external magnetic activation. As a matter of fact, the bigger the internal torque on the $\mathrm{IPM}_{3}$, the more robust is the closure of the gates. However, it implies also having a bigger external magnet for remote activation. Hence, first the IPMs were dimensioned and their relative distances computed. Secondly, the magnetic field able to overcome the torques on the IPMs during the opening and brushing phase was computed. Finally, the EPM able to provide the computed field was selected, and the operational distances from the capsule, for each working phase, were assessed. The optimal volume, shape and type of magnetization of the EPM were investigated to provide an almost uniform magnetic field at the capsule site. To this end, two models were used for solving the systems of equations: one analytical and one numerical. The analytical modelling is an implementation of the charge and current model for approximating the magnetic field flux density, interaction forces and torque deriving from a magnetic source [11]-[13]. The framework was developed using Matlab (Mathworks). Whereas the numerical model involves the solution of the magnetostatic equations using the Finite Element Method (FEM) with the software COMSOL Multiphysics $^{\circledR}$ (Stockholm, Sweden). Lastly, for a final validation of the modelling framework, benchtop experiments were conducted with test magnets (Section V). 


\section{B. Internal torques between IPMs}

The dimensions of the internal magnets were maximized, to increase the torque generated by the EPM on the IPMs. As a matter of fact, bigger magnets experience higher torques and forces under a fixed magnetic field. The final values are reported in Table 1. All the magnets are $\mathrm{NdFeB}$ with N52 magnetization grade, the highest available (i.e., $\mathrm{Br}=1.45 \mathrm{~T}$ ).

As a second step, a set of simulations was conducted to investigate the optimal solution for locating the magnets inside the capsule. To this end, the internal torques, for the three working phases were computed varying the length of the capsule from 26.5 to $30.5 \mathrm{~mm}$, with steps of $1 \mathrm{~mm}$. This specific range falls between the length of the Pillcam ${ }^{\mathrm{TM}}$ SB3 (i.e., $26.2 \mathrm{~mm}$ ) and the Pillcam ${ }^{\mathrm{TM}}$ Colon (i.e., $32.3 \mathrm{~mm}$ ), leaving few $\mathrm{mm}$ from the maximum length to account for any addition external covers or sheaths that might increase the final size. Meanwhile, the surface-surface distance between

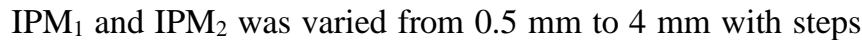
of $0.5 \mathrm{~mm}$. The results are shown in Fig. 4. As the best tradeoff between stability (low internal torques cause the capsule to open too easily, even when not desired) and remote activation, the capsule dimensions were established, in the first place, of $28.5 \mathrm{~mm}$, and maximum distance between IPMs 1 and 2 (i.e., $d=3 \mathrm{~mm}$ ). Accordingly, the torque needed to rotate the $\mathrm{IPM}_{3}$ during the opening phase is $3.7 \mathrm{mNm}$, and to rotate IPMs 2 and 4 during the brushing is $6.05 \mathrm{mNm}$ (solution computed analytically). These values are provided by an external uniform magnetic fluid density of at least $B=30 \mathrm{mT}$ (opening) and $B=75 \mathrm{mT}$ (brushing), as resulting from a second set of simulations.

\section{External torques induced by an EPM on the IPMs}

The first step towards the selection of the EPM was choosing its optimal shape and the type of magnetization, at a fixed volume, inducing the highest torques on $\mathrm{IPM}_{2}$ and $\mathrm{IPM}_{3}$. Following, the volume can be scaled to allow the proper triggering of the opening and brushing phase, at distances compatible with the anatomy of the patient. As a result of an initial pilot experimentation, an EPM volume of around $1000 \mathrm{~cm}^{3}$ is needed to activate IPMs 2 and 4 at a distance of $100 \mathrm{~mm}$, which is the usual range used for endoscopic magnetic locomotion [10]. Hence, fixing the volume of the EPM to $1000 \mathrm{~cm}^{3}$, the torque on $\mathrm{IPM}_{2}$ induced by several cylinders with axial and diametrical

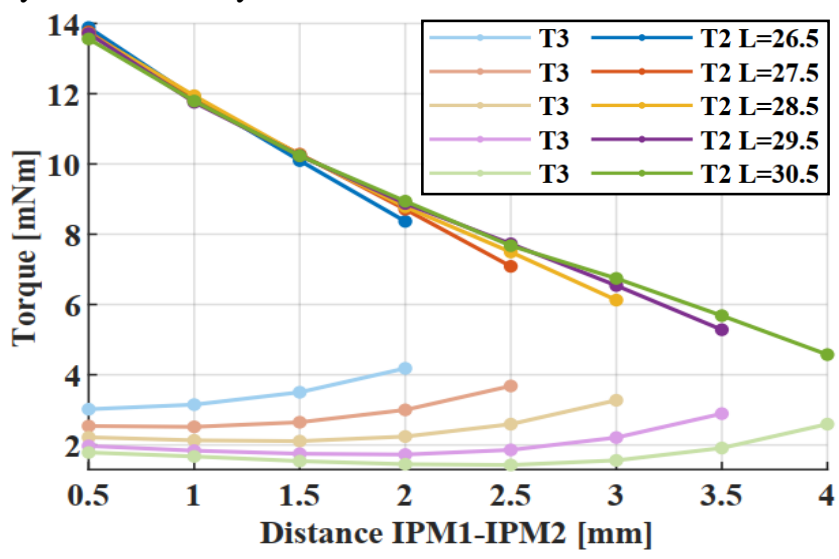

Figure 4. Peak torque curves of $\mathrm{IPM}_{2}$ and $\mathrm{IPM}_{3}$ varying the length of the capsule $(\mathrm{L}[\mathrm{mm}])$ and the distance between $\mathrm{IPM}_{1}$ and $\mathrm{IPM}_{2}$ (results of the analytical model). These values are those that need to be overcome by the EPM to open the gate $\left(\tau_{3}\right)$ and brush the mucosa $\left(\tau_{2}\right)$. magnetization, and cubes, with different sizes ratio were computed (EPMs $\mathrm{Br}=1.45 \mathrm{~T}$ ), at variable distances. The results are shown in Fig. 5, and highlight that the highest torque is provided by the axially-magnetized cylinder with dimensions: $D=15 \mathrm{~cm} L=5,6 \mathrm{~cm}$.

With these dimensions, the EPM overcome the $\mathrm{IPM}_{2}$ peak torque at a distance of $100 \mathrm{~mm}$ from the capsule (surface-tosurface) and the $\mathrm{IPM}_{3}$ peak torque at $160 \mathrm{~mm}$. Hence, referring to Fig. 3, $d_{2}$ could be set to $160 \mathrm{~mm}$ (opening), and $d_{3}$ to $100 \mathrm{~mm}$ (brushing). These values fall in the range usually considered as the average distance between an endoscopic capsule and the skin, for people with a normal body mass index (BMI) [10]. However, giving the scalability of the framework and the lack of any restriction on the size of the external actuator, the EPM volume might be increased in the future to be compatible with patients having higher BMI.

\section{V.EXPERIMENTAL VALIDATION}

To assess the magnetic models and verify the predicted torques, a series of four benchtop experiments were conducted (named A, B, C, D). Since capsule's selected magnets require a custom production, high cost and long delivery times, easy-to-acquire and commercially-available magnetic cylinders have been used for testing the model. Each test required the use of a robotic arm (RV-3SB, Mitsubishi Corp., Tokyo, Japan) for high precision regulation of the distances. A triaxial force/torque sensor, (Nano 17-E, ATI Industrial Automation, U.S.) was used in tests A and B for measuring the torques. Each time, the five readings from the sensor were acquired, and its average value was used as a reference. For all the four experiments, the supports were either 3D printed in ABS, or manufactured with Teflon ${ }^{\circledR}$ and aluminum to avoid magnetic interferences. The screws used to fix the supports and the shaft were non-ferromagnetic (i.e.,

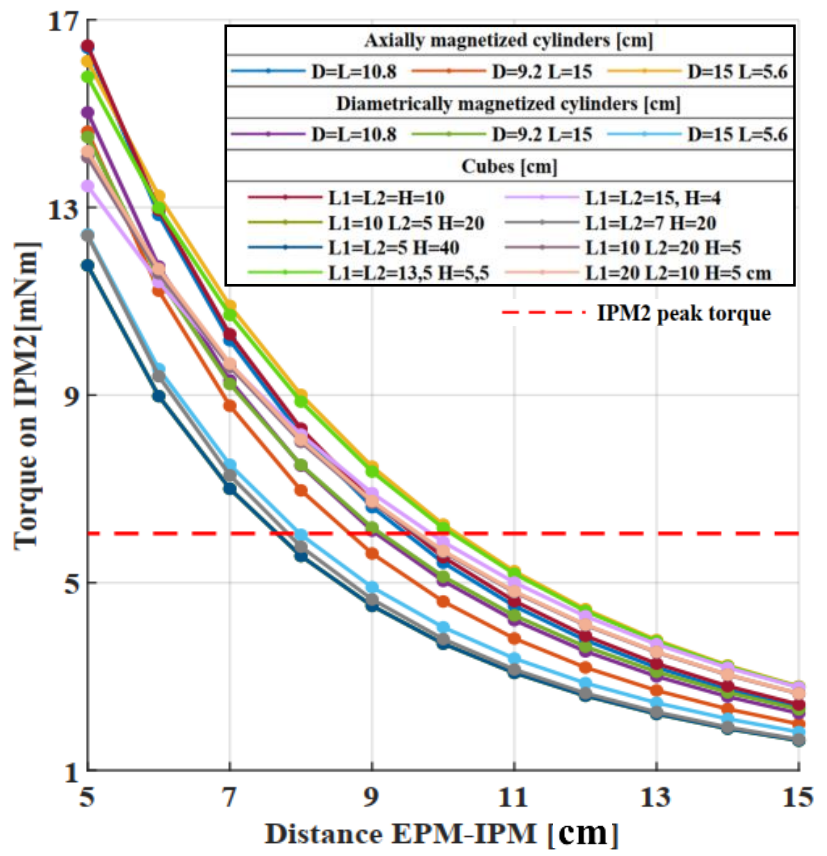

Figure 5. Torques induced on $\mathrm{IPM}_{2}$ by different EPM with fixed volume of $1000 \mathrm{~cm}^{3}$, at variable distances IPM-EPM (results of the analytical model). The EPMs investigated are axially magnetized cylinders, diametrically magnetize cylinders, and cubes. The red line shows the peak torque for $\mathrm{IPM}_{2}$, which when reached, allow to rotate the magnet (brushing). 
aluminum, Teflon ${ }^{\circledR}$ ). Each test was repeated five times, and the results obtained were compared with the predicted values of both the analytical and numerical models.

\section{A. Test A}

A first step in assessing the model was to validate the estimated torque between two concentric cylindrical magnets, varying their relative distance and angle. This configuration is the one used for estimating the internal torques between the IPMs. To this end, two identical hollow cylinders with diametrical magnetization $\left(\mathrm{NdFeB} 35 \mathrm{H}, B_{r}=1.17 \mathrm{~T}, L=6 \mathrm{~mm}\right.$, $D=8 \mathrm{~mm}$ and $d=2 \mathrm{~mm}$ ) were used. As shown in Fig. 6.a, one magnet was fixed to the benchtop, while the other one was attached to the load cell through a connector made in aluminum, long enough to avoid any interferences of the sensor with the magnetic field. The load cell was then attached to the robotic arm, to control the magnet position. In a first test (Test A.1), the two cylinders were placed one in front of the other, with a $90^{\circ}$ mismatch between their polarization vectors, and a surface-to-surface distance ranging from 5 to $57 \mathrm{~mm}$, considering $1 \mathrm{~mm}$ steps.

In a second test (Test A.2), the distance between the cylinders was fixed to $10 \mathrm{~mm}$, and the magnet attached to the robot was rotated from 0 to $360^{\circ}$, with steps of $30^{\circ}$ each. In both cases, for each step, the axial torque was recorded and noted.

\section{B. Test B}

The second experimental set-up was designed to validate the torque estimated between one IPM and the EPM. As in the previous experiment, one diametrically magnetized cylinder $\left(\mathrm{NdFeB} 35 \mathrm{H}, B_{r}=1.17 \mathrm{~T}, L=6 \mathrm{~mm}, D=8 \mathrm{~mm}\right.$ and $d=2 \mathrm{~mm}$ ) was attached to a load cell, connected to the robotic arm. However, in this case, a bigger cylindrical magnet (NdFeB N35, $B_{r}=1.35 \mathrm{~T}, D=50 \mathrm{~mm}$ and $L=25 \mathrm{~mm}$ ), with axial magnetization, was fixed to the benchtop. In the first test (Test B.1), the magnet attached to the robotic arm was progressively moved towards the fixed magnet from a distance of 34 to $12 \mathrm{~mm}$, with $1 \mathrm{~mm}$ steps. Meanwhile, the angle between the two magnetization vectors was fixed to $90^{\circ}$ as shown in Fig. 6.b. Whereas, in the second test (Test B.2), the distance surface-to-surface between the two components was fixed to $50 \mathrm{~mm}$, and the IPM was rotated around its main axis from an angle of $0^{\circ}$ to $180^{\circ}$, with steps of $30^{\circ}$. In both cases, at each step, the torque was recorded by the load cell and noted down.

a)

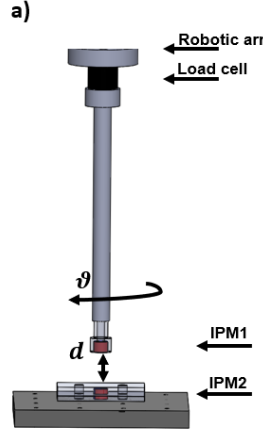

b)

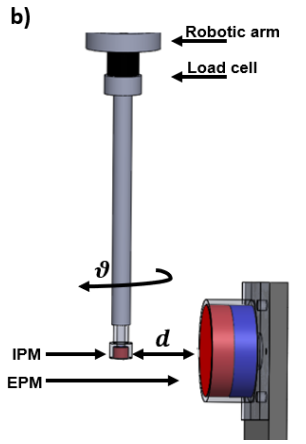

\section{C.Test C}

The third set of experiments aimed at validating the magnetic torsional spring model, and the estimated operational distance of the EPM. In this case, the two cylindrical magnets used in the first test (i.e., $B_{r}=1.17 \mathrm{~T}, L=6$ $\mathrm{mm}, D=8 \mathrm{~mm}$ and $d=2 \mathrm{~mm}$ ) were mounted on a shaft, which was fixed through a support to the benchtop (Fig. 6.c). One magnet was fixed, while the other (free to rotate), was placed at a surface-to-surface distance of $6.5 \mathrm{~mm}$ from the first one. A disk made of a low friction material (i.e., Teflon ${ }^{\circledR}$ ) was used as a spacer for the magnets. The previously introduced axially-magnetized cylinder, was attached to the robotic arm using a non-ferromagnetic connector $\left(B_{r}=1.35 \mathrm{~T}, D=50 \mathrm{~mm}\right.$ and $L=25 \mathrm{~mm}$ ). Starting from a distance of $100 \mathrm{~mm}$ from the IPMs, the EPM was progressively moved forward. The distance IPM-EPM at which the magnet free to rotate made a turn of $180^{\circ}$ was noted.

\section{D.Test D}

Finally, the last experiment was designed to test the whole system. In this case, five different diametrically magnetized hollow cylinders, resembling those sized for the capsule, were mounted on a support. The most external magnets (in Fig. 6.d IPMs 1 and 5) were fixed (NdFeB 35H, $B_{r}=1.17 \mathrm{~T}, L=6 \mathrm{~mm}, D=8 \mathrm{~mm}$ and $\left.d=2 \mathrm{~mm}\right)$, as in the endoscopic capsule. Two additional hollow cylinders $\left(\mathrm{Sm} 2 \mathrm{Co} 17 \mathrm{YXG} 30, B_{r}=1.08 \mathrm{~T}, L=2 \mathrm{~mm}, D=8 \mathrm{~mm}, d=2\right.$ $\mathrm{mm}$ ), reproducing IPMs 2 and 4, were spaced (surface-tosurface) $2 \mathrm{~mm}$ apart, from the closest fixed magnet, and let free to rotate. Finally, a thin cylinder (Sm2Co17 YXG30, $B_{r}=1.08 \mathrm{~T}, L=4 \mathrm{~mm}, D=12 \mathrm{~mm}$ and $d=8.5 \mathrm{~mm}$ ) was mounted on a cylindrical support, fixed to the shaft. This magnet, mimicking $\mathrm{IPM}_{3}$, was let free to rotate, and was spaced $2 \mathrm{~mm}$ respectively from magnets 2 and 4 (see Fig. 6.d). All the spacer and the support were built in Teflon ${ }^{\circledR}$, to minimize the contact friction. The previously mentioned axiallymagnetized cylinder $\left(B_{r}=1.35 \mathrm{~T}, D=50 \mathrm{~mm}\right.$ and $\left.L=25 \mathrm{~mm}\right)$, was attached to the robotic arm, with its surface centering the magnets mounted on the support. The cylinder was moved from a distance of $130 \mathrm{~mm}$ towards the IPMs. The points in which magnets 2 and 4 and magnet 3 turned was recorded.

\section{E. Results}

The aim of the experiments was to validate the modelled torques, forces and EPM operational distances. The results are summarized in Table 2. To this end, Test A and B
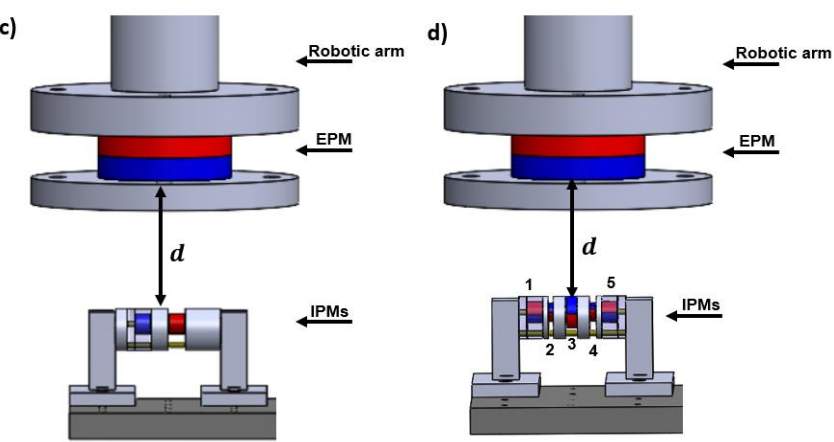

Figure 6. a) Experimental setup to validate the internal torque between two IPMs varying their relative distance d and the angle $\theta$; b) experimental setup to validate the torque induced by the EPM on a IPM varying their relative distance d and the angle $\theta$; $c$ ) experimental setup to validate the distance at which the EPM triggers the magnetic torsional spring, and allows the rotation of an IPM; d) experimental setup to validate the distances at which the EPM triggers the rotation of $\mathrm{IPM}_{3}$ (opening) and $\mathrm{IPM}_{2.4}$ (brushing). 
provided good results, having the experimental torque curves overlapping with the estimated ones (see Fig. 7); for Test A.2, FEM outperformed the analytical solution. Regarding Test $\mathrm{C}$ a slight mismatch between simulated and real results was reported. This is probably due to the non-perfect alignment between the EPM and IPM center, which induces additional torques that contribute to the rotation of the IPM (happening at a slight further distance IPM-EPM than the simulated one). Finally, in Test D, both $\mathrm{IPM}_{3}$ and $\mathrm{IPM}_{2,4}$ rotated when the EPM was closer to the estimated operational distance. The reason for this observed behavior comes from an additional resistive factor which contribute to the overall axial torque of the IPM. As a matter of fact, while rotating under the influence of the EPM, the IPMs experience internal attractive/resistive magnetic forces and slight torques on the other two axes. These, together with the contact friction, increase the overall mechanical torque on $\mathrm{IPM}_{3}$ of a $30 \%$. Whereas, for $\mathrm{IPM}_{2,4}$, it doubles the resistive peak torque.

\section{DISCUSSION AND CONCLUSION}

In this paper, we present a novel magnetic actuation system of an ingestible capsule designed for enabling the collection of microbiota samples throughout the whole GI tract. The system allows in situ sampling of the microbiota, and remote activation with an external permanent magnet. Localization of the system is possible, performing triangulation of the magnetic flux density measured with external Hall effect sensors (e.g., asking the patient to wear a belt with hall effect sensors) [14]. However, its development is out of the scope of this paper. The passive nature of the magnets, replacing standard electrical components, intrinsically makes the system safer and easier to translate in the clinical practice. The actuation system, based on multiple magnetic torsional springs, was dimensioned through a scalable framework, based on numerical and analytical simulations. The validity of the computational model and the functions of the actuation system were evaluated experimentally with a series of benchtop tests. Accordingly, a scaled set-up assessed the validity of the computed torques and verified the overall operations of the system. To this end, the experimental platform included a set of permanent magnets with slight bigger size to those designed for the final prototype. The tests allowed to estimate a resistive mechanical factor which contributes to the torque on the IPMs for a second-round design and final implementation.

As a final step, considering the discovery of the corrective parameter through the experiments, the prototype
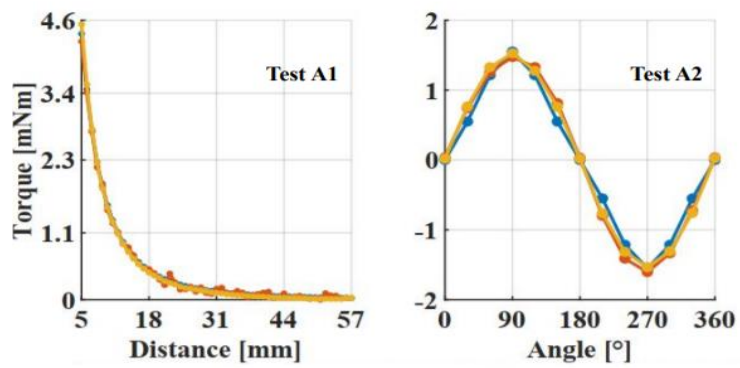

TABLE 2. SUMMARY OF RESULTS OF TEST A AND TEST B

\begin{tabular}{|c|c|c|c|}
\hline \multirow[b]{2}{*}{ Test } & \multicolumn{2}{|c|}{ Estimated torque error (Mean \pm SD) } & \\
\hline & $\begin{array}{c}\text { Analytical vs } \\
\text { experimental data }\end{array}$ & $\begin{array}{c}\text { Numerical vs } \\
\text { experimental data }\end{array}$ & \\
\hline A.1 & $0.02 \pm 0.02 \mathrm{mNm}$ & $0.05 \pm 0.04 \mathrm{mNm}$ & \\
\hline A.2 & $0.1 \pm 0.04 \mathrm{mNm}$ & $0.08 \pm 0.02 \mathrm{mNm}$ & \\
\hline B.1 & $0.13 \pm 0.05 \mathrm{mNm}$ & $0.21 \pm 0.12 \mathrm{mNm}$ & \\
\hline \multirow[t]{3}{*}{ B.2 } & $0.02 \pm 0.02 \mathrm{mNm}$ & $0.03 \pm 0.01 \mathrm{mNm}$ & \\
\hline & \multicolumn{2}{|c|}{ Estimated distance } & Experimental \\
\hline & Analytical model & Numerical model & $\begin{array}{c}\text { distance } \\
(\text { Mean } \pm \text { SD) }\end{array}$ \\
\hline$C$ & $77 \mathrm{~mm}(\Delta=5 \mathrm{~mm})$ & $76 \mathrm{~mm}(\Delta=6 \mathrm{~mm})$ & $82 \pm 1.4 \mathrm{~mm}$ \\
\hline$D$ & $\begin{array}{l}\text { Open: } 105 \mathrm{~mm} \\
\text { Brush: } 32 \mathrm{~mm}\end{array}$ & $\begin{array}{l}\text { Open: } 110 \mathrm{~mm} \\
\text { Brush: } 32 \mathrm{~mm}\end{array}$ & $\begin{array}{l}\text { Open: } 95 \pm 4.5 \mathrm{~mm} \\
\text { Brush: } 18 \pm 3 \mathrm{~mm}\end{array}$ \\
\hline
\end{tabular}

design is modified. Accordingly, the final capsule length is set to $30.5 \mathrm{~mm}$. Doing so, the pure magnetic peak torque on $\mathrm{IMP}_{2,3,4}$ is decreased. Following, the size of the EPM is increased to $L=73 \mathrm{~mm}$ and $D=195 \mathrm{~mm}$ : to allow the activation despite the additional resistive contribution on the IPMs torques. In this way the new EPM operational distances are $d_{2}=200 \mathrm{~mm}$ (i.e., opening) and $d_{3}=100 \mathrm{~mm}$ (i.e., brushing), still compatible with operating distances. As a matter of fact, the final dimensioning is made in order to keep the minimum distance capsule (IPMs)-EPM (i.e., $d_{3}$ ) to $100 \mathrm{~mm}$, which is a reasonable estimation of the average distance between the skin and the bowel [10]. In this way, the EPM dimensions were modified maintaining the shape, type of magnetization, and the dimensional ratio between the diameter and the height, set in Section IV and considered optimal. With a final volume of $2179 \mathrm{~cm}^{3}$, and a weight of $16.3 \mathrm{~kg}$, the EPM might be fixed to a supportive arm (e.g., Martin's Arm), and manipulated by hands from the clinician.

The design parameters, derived from the validated computational models and the benchtop experiments, will enable the authors to build the final capsule prototype. Hence, all the components will be mounted inside a biocompatible case and a set of future experiments will aim at assessing the ability of the designed device to collect and store microbiota samples, in ex-vivo and in-vivo scenarios. Foreseen challenges include ensuring the sealing of the capsule, to avoid any contamination of the samples, but keeping the friction forces low. Possible solutions might involve a combination of different technologies, e.g., siliconbased plugs, expanding hydrogels, eccentric rotational mechanism and external enteric coverings.
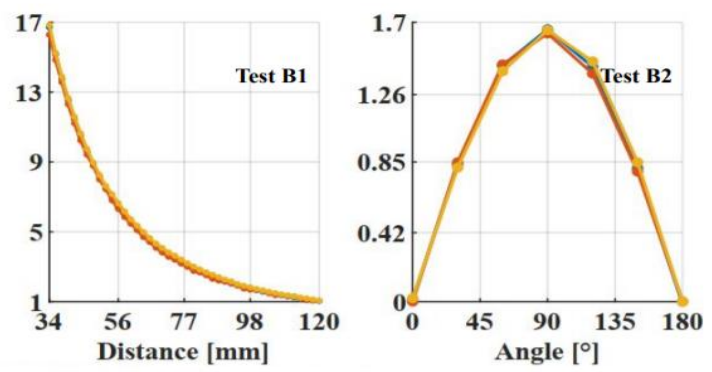

Experimental

Figure 7. Torque curves obtained from tests A and B: comparison of experimental results. with the analvtical and numerical models. 


\section{REFERENCES}

[1] E. N. Janoff, "The microbiome and human disease pathogenesis: how do you do what you do to me ...?," Translational Research, vol. 179. Mosby Inc., pp. 1-6, Jan. 01, 2017, doi: 10.1016/j.trsl.2016.10.007.

[2] L. K. Ursell, J. L. Metcalf, L. W. Parfrey, and R. Knight, "Defining the human microbiome," Nutr. Rev., vol. 70, no. SUPPL. 1, p. S38, Aug. 2012, doi: 10.1111/j.1753-4887.2012.00493.x.

[3] E. Thursby and N. Juge, "Introduction to the human gut microbiota," Biochemical Journal, vol. 474, no. 11. Portland Press Ltd, pp. 1823-1836, Jun. 01, 2017, doi: 10.1042/BCJ20160510.

[4] G. Ciuti et al., "Frontiers of robotic endoscopic capsules: a review," Journal of Micro-Bio Robotics, vol. 11, no. 1-4. Springer Verlag, Jun. 01, 2016, doi: 10.1007/s12213-016-0087-x.

[5] M. Ben Salem, G. Aiche, L. Rubbert, P. Renaud, and Y. Haddab, "Design of a Microbiota Sampling Capsule using 3D-Printed Bistable Mechanism," in Proceedings of the Annual International Conference of the IEEE Engineering in Medicine and Biology Society, EMBS, Oct. 2018, vol. 2018-July, pp. 48684871, doi: 10.1109/EMBC.2018.8513141.

[6] H. Rezaei Nejad et al., "Ingestible Osmotic Pill for In Vivo Sampling of Gut Microbiomes," Adv. Intell. Syst., vol. 1, no. 5, p. 1900053, Sep. 2019, doi: 10.1002/aisy.201900053.

[7] "PillCam ${ }^{\mathrm{TM}}$ SB 3 System | Medtronic." https://www.medtronic.com/covidien/enus/products/capsule-endoscopy/pillcam-sb-3system.html (accessed Oct. 18, 2020).

[8] G. Li et al., "Diversity of duodenal and rectal microbiota in biopsy tissues and luminal contents in healthy volunteers," J. Microbiol. Biotechnol., vol. 25, no. 7, pp. 1136-1145, 2015, doi: 10.4014/jmb.1412.12047.

[9] M. J. Claesson, A. G. Clooney, and P. W. O'Toole, "A clinician's guide to microbiome analysis," Nature Reviews Gastroenterology and Hepatology, vol. 14, no. 10. Nature Publishing Group, pp. 585595, Oct. 01, 2017, doi: 10.1038/nrgastro.2017.97.

[10] M. Simi, G. Gerboni, A. Menciassi, and P. Valdastri, "Magnetic torsion spring mechanism for a wireless biopsy capsule," J. Med. Devices, Trans. ASME, vol. 7, no. 4, Sep. 2013, doi: 10.1115/1.4025185.

[11] Permanent Magnet and Electromechanical Devices. Elsevier, 2001.

[12] E. P. Furlani, S. Reznik, and W. Janson, "A ThreeDimensional Field Solution for Bipolar Cylinders," IEEE Trans. Magn., vol. 30, no. 5, pp. 2916-2919, 1994, doi: 10.1109/20.312547.

[13] J. Li et al., "Magnetically-driven medical robots: An analytical magnetic model for endoscopic capsules design," J. Magn. Magn. Mater., vol. 452, pp. 278287, Apr. 2018, doi: 10.1016/j.jmmm.2017.12.085.

[14] F. Bianchi et al., "Expert Review of Medical Devices Localization strategies for robotic endoscopic capsules: a review Localization strategies for robotic endoscopic capsules: a review," 2019, doi:

10.1080/17434440.2019.1608182. 